\title{
Heart Murmur Classification with Feature Selection
}

\author{
D. Kumar, P. Carvalho, M. Antunes ${ }^{\dagger}$, R. P. Paiva, J. Henriques \\ Centre for Informatics and Systems, University of Coimbra, Portugal \\ \{dinesh, carvalho, ruipedro, jh\}@dei.uc.pt \\ ${ }^{\dagger}$ Cardiothoracic Surgery Centre, University Hospital of Coimbra, Portugal \\ antunes.cct.huc@sapo.pt
}

\begin{abstract}
Heart sounds entail crucial heart function information. In conditions of heart abnormalities, such as valve dysfunctions and rapid blood flow, additional sounds are heard in regular heart sounds, which can be employed in pathology diagnosis. These additional sounds, or so-called murmurs, show different characteristics with respect to cardiovascular heart diseases, namely heart valve disorders. In this paper, we present a method of heart murmur classification composed by three basic steps: feature extraction, feature selection, and classification using a nonlinear classifier. A new set of 17 features extracted in the time, frequency and in the state space domain is suggested. The features applied for murmur classification are selected using the floating sequential forward method (SFFS). Using this approach, the original set of $\mathbf{1 7}$ features is reduced to 10 features. The classification results achieved using the proposed method are compared on a common database with the classification results obtained using the feature sets proposed in two well-known state of the art methods for murmur classification. The achieved results suggest that the proposed method achieves slightly better results using a smaller feature set.
\end{abstract}

keywords: heart murmur, heart sound, SVM, feature selection.

\section{INTRODUCTION}

Auscultation is the preferred method for heart valve disorders diagnosis [1]. Therefore, a system including sensor based heart sound recording and subsequent analysis of the phonocardiogram with potential key features may help physicians to diagnose valve related disorders. In our work, two main issues regarding heart sound analysis for murmur classfication: the first one is to identify starting and stop points of the murmur which are, in most cases, complimentary to the S1 and S2 sound start and stop points, and the second one is to classify the murmur into its category (e.g. mitral regurgitation, aortic stenosis, etc). The problem of finding murmur boundaries is solved using an in-house method of decomposition and simplicity [2]. As for murmur recognition, the main topic of the present paper, this task is tackled as a pattern recognition problem that resorts to the extraction of discriminative features to classify murmurs into their categories.

The main challenge in the context of heart murmur classification is feature extraction. In the past few years many researchers have attempted to solve this problem, having achieved significant results. Namely, a feedforward neural network for murmur detection and two Learning Vector Quantization (LVQ) networks were employed for murmur classification in [3]. The murmur classification system is designed to categorize the detected murmur into 3 common types: Mitral Stenosis, Aortic insufficiency and Mitral insufficiency. Two LVQ networks were trained and tested using the level 4 and level 5 discrete wavelet transform approximations of the heart signals. The accuracy rates of the detection and classification subsystems were $73.12 \%$ and $67.05 \%$, respectively. In [4], a binary hierarchical three classifiers murmurs into atrial flutter, aortic regurgitation, aortic stenosis and mitral stenosis. The author used the morphological characteristics of power spectral distribution of the heart sound in the frequency domain as input features. The reported murmur detection accuracy was $86.88 \%$ for atrial fibrillation sounds, $89.98 \%$ for sounds exhibiting aortic valvular disorders. In [5], a feed-forward neural network classifier was employed. There, input features were extracted from the spectrogram of averaged heart cycles. The system attained $85 \%$ accuracy in the discrimination between normal sounds, sounds with aortic stenosis and sounds with aortic regurgitation. The main limitation of the previous approaches is that they were evaluated on individual rather than benchmark databases, making it difficult to perform a fair comparison between different methods.

In this paper, a new set of features, extracted from time, frequency and state space domains of signal transformation, is introduced. The extracted features are evaluated with the sequential floating feature selection (SFFS) method [6]. This process reduces the number of features in the feature set by selecting discriminative features. After feature selection, the selected features are used to train a nonlinear support vector machine (SVM) classifier.

In order to perform a comparative study between the proposed method and the state-of-the-art, two widely cited methods are implemented. The first approach, by Olmez et al. [7] extracts, 32 features from a wavelet decomposed heart sound, and uses all the features to construct a nonlinear classifier. The second method, by Ahlstrom et al. [8], resorts to 207 features that are extracted from a vast area of signal processing and statistical methodologies. In order to check the discriminative value of the proposed set of features, the achieved results are also compared against the classification results achieved with the best feature set selected by SFFS among all considered features in this work, i.e. the proposed feature set and the feature sets proposed by Olmez et al. and by Ahlstrom et al..

The paper is organized as follows: in the second section, feature extraction and selection methods are explained, in 
the third section results and discussion are presented and in the final section some conclusions are withdrawn.

\section{Methodology}

In order to implement feature extraction in each of the aforementioned approaches, the key step is to identify heart cycles. From each heart cycle features are extracted and then utilized to construct a classifier. To this end, heart beats are identified using the authors' previous work regarding S1 and S2 sounds detection [9]. The next step is to extract features from every heart cycle and to recognize the type of murmur using a nonlinear classifier (SVM, in this case). In the first subsection, feature extraction in various analysis domains, such as time, frequency, time-frequency, nonlinear high order statistics, state space, etc. is performed.

\section{A. Feature extraction}

The heart sound will be denoted as $x(n)$, where $n=1,2 \ldots N$, and $N$ is the number of samples in the time period (heart cycle). All the features are normalized to zero mean and unit standard deviation.

\section{Feature Set 1}

This is the new set of features proposed by the authors, which includes time, frequency and statistical or phase space features. These features are described in the following paragraphs.

1) Time domain features: Some features, such as intensity, frequency location over time and shape, are computed in the time domain. These characteristics are obtained by computing loudness (loudness 1), jitters (jitters 1), zero-crossing rate $(\mathrm{zcr} 1)$, and transition ratio (transition ratio 1) [10] of the systolic period according to equations, in (1-4) below.

$$
\text { loudness }=\sqrt{\frac{\sum_{n=n^{i}}^{n^{e}} x(n)^{2}}{n^{e}-n^{i}}}
$$

where $n^{i}$ is starting sample in murmur segment and $n^{e}$ is the stop sample. As mentioned above, the loudness feature is normalized and, hence, independent of the amplifier's gain.

$$
\text { Jitter }=\frac{\sum_{m=2}^{P-1} 2 T_{p}(m)-T_{p}(m-1)-T_{p}(m+1)}{\sum_{m=2}^{P-1} T_{p}(m)}
$$

where $P$ is the total number of peaks in the autocorrelation function, and $T_{p}(m), m=1,2, \ldots . P$, is the time separation between the $m^{t h}$ and the $(m+2)^{t h}$ local maxima of the autocorrelation of the heart sound signal.

$$
\begin{gathered}
z c r=\frac{1}{n^{e}-n^{i}} \sum_{j=n^{i}}^{n^{e}}|\operatorname{sgn}(x(j))-\operatorname{sgn}(x(j-1))| \\
\text { transition ratio }=\frac{T_{a s c}}{T_{d s c}},
\end{gathered}
$$

where $T_{\text {asc }}$ is the transition time taken from the first minimum of the energy curve to the maximum energy, and $T_{d s c}$ is the time interval from the energy maximum to the last subsequent minimum energy.

(2) Frequency domain features capture characteristics of the signal's timbre and morphology. In order to compute those features, the power spectrum of the signal is computed resorting to the periodogram. 10 frequency-domain features are extracted, as explained below.

Spectral power: Spectral power is computed through the periodogram via summation over frequency. Since the power of murmurs spreads across various frequency regions $(0-400 \mathrm{~Hz})$, in order to examine the dominance of spectral power at specific frequencies, spectral power is computed in four frequency bands: $0-100 \mathrm{~Hz} ; 100-200 \mathrm{~Hz}$, $200-300 \mathrm{~Hz}$, and $300-400 \mathrm{~Hz}$. Hence, 4 features (spectral power 1-4), corresponding to the power in each of 4 bands, are computed by summing over frequency.

Spectral power-based features: From the power spectrum above, the following features are extracted, which aim to capture each murmur's morphology and shape: centroid (spec centroid 1), flux (spec fluxl), skewness (spec skewness 1) and kurtosis (spec kurtosis 1). In addition, two fundamental frequency features are extracted (fundamental frequency 1-2) [11].

(3) Statistical domain features: The distribution and scattering of samples in the murmur is observed with recourse to histograms and phase space. The following 3 features are computed:

Skewness and Kurtosis: Two measures, skewness (stat skewness 1) and kurtosis (stat kurtosis 1), are computed through the histogram of the heart murmur segment.

Chaos: The maximum of Lyapunov exponents (Lyapunovexponents 1), from [10], is taken as the quantifier of the degree of chaos in the murmur segments.

\section{Feature Set 2}

This set of features is created using Olmez et al. [7] method of feature extraction. The authors perform wavelet decomposition to represent the heart sounds in different frequency bands. The 2 nd details coefficients, $\mathrm{d} 2$, of the Discrete Wavelet Transform (DWT) is utilized to compute its power in the following way: the $\mathrm{d} 2$ is split into 32 sub-windows with 128 discrete samples each. To construct a feature matrix (PoS 1-32), the power of every split signal for the 32 sub-windows is computed.

\section{Feature Set 3}

Feature set 3 is comprised with the feature set suggested by 
Ahlstorm et al. [8]. These authors extracted an extensive set of 207 features using several signal processing and mathematical techniques. In time domain, 9 values of Shannon energy (Shannon energy 1-9) are calculated. In the time-frequency domain 11 features are extracted using the Shannon entropy of the 10th level wavelet detail coefficients (WT entropy 1-11), 9 energy measures are obtained from the high frequency components of the signal using 6th level details wavelet coefficients (WT detail 1-9), 16 features are obtained from the S-transform (ST map 1-16), 8 eigenvalues are calculated from singular value decomposition of the S-transform, 40 features are extracted from two left and two right eigenvectors and their corresponding histograms are also included (Eigenvalues 1-8, Eigenfrequency 1 1-10, Eigenfrequency 2 1-10, Eigentime1 1-10, Eigentime2, 1-10). In the category of nonlinear and chaos based or state space based features, the applied feature set includes: 16 discretized equally sized triangles from non-redundant region of the bispectrum (HOS 1-16), 40 features from Gaussian mixture model of the reconstructed space of the heart cycle (GMMx cycle 1-40), 40 from murmur (GMMx murmur 1-40), 8 features obtained using the variance fractal dimension (VFD 1-8) and 10 features from recurrence quantification analysis (RQA 1-10). The details regarding the calculation of each of the mentioned features can be found in [8].

\section{Feature Set 4}

In order to find the best features among all the extracted features from the three aforementioned sets, as well as to determine the impacts of introduced features (from set 1), a feature set 4 is created. This set of features is the superset of the previous three sets of features.

\section{B. Feature selection and classification}

In order to decrease the classifier's complexity, feature selection might be applied to select among the most discriminative features. In the present work, a subset of features are selected for the new feature set (feature set 1) reported in section II.a using Paudil's sequential floating forward selection (SFFS) strategy. This method selects or rejects features based on the error estimate of a 1-nearest neighbor leave-one-out classifier. In our approach, the selection is performed with the toolbox developed by Ververidis [12].

It should be mentioned that a similar strategy is suggested by Ahlstrom et al. in order to reduce the original set of 207 features to a more manageable subset of 14 features. In the provided comparisons this feature set is identified by feature set 3. The features which comprise this set are the ones listed in the original work by Ahlstrom and colleagues. Regarding Olmez et al. algorithm, no feature selection has been performed because the complete set composed by 32 features are applied by these authors. Finally, the aforementioned feature selection strategy was applied to all the features mentioned in the previous subsection.

Regarding the classification stage, for each of these feature sets, a SVM classifier was trained and applied. A method based on the "one-against-one" (OAO) binary classification approach was chosen using a cubic polynomial kernel function.

\section{RESUlts AND Discussions}

Heart sounds containing murmurs were collected from the Cardiothoracic Surgery Center of the University Hospital of Coimbra. Acquisition was performed with an electronic stethoscope from Meditron. The stethoscope presents excellent signal-to-noise ratio characteristic and an extended frequency range $(20-20,000 \mathrm{~Hz})$. Sound samples were recorded for the maximum duration of one minute, using a 16-bit $\mathrm{ADC}$ at $44.1 \mathrm{kHz}$ sampling rate. A total of 81 heart sound clips with murmurs, corresponding to 2047 beats, were collected from 51 patients. The main biometric characteristics of the population were: $\mathrm{BMI}=25.41 \pm 2.16$; age $=64.65 \pm 8.64$; number of males $=48$; number of females $=3$.

In the classification stage, sounds classified as murmur are categorized into the following 7 classes: Aortic Regurgitation (AR), Aortic Stenosis (AS), Mitral Regurgitation (MR), Pulmonary Regurgitation (PR), Pulmonary Stenosis (PS), Subaortic Stenosis + Ventricular Septal Defect (SAS+VSD), Systolic Ejection (SE).

TABLE I

SELECTED FEATURES IN THE FEATURE SETS.

\begin{tabular}{|c|c|}
\hline \multicolumn{2}{|l|}{ Feature Set I } \\
\hline loudness 1 & $z c r 1$ \\
\hline transition ratiol & spectral power 2 \\
\hline fundamental frequency 1 & spectral shape 1 \\
\hline spectral power 3 & flux 1 \\
\hline stat skewness 1 & Lyapunovexponent 1 \\
\hline \multicolumn{2}{|l|}{ Feature Set 2} \\
\hline PoS 1-32 & no selection, kept as in original work \\
\hline \multicolumn{2}{|l|}{ Feature Set 3} \\
\hline WT detail 7 & DFD 8 \\
\hline Shannon energy 5 & Shannon energy 6 \\
\hline GMM cycle 5 & Shannon energy 4 \\
\hline GMM murmur 5 & Eigenfrequencyl 2 \\
\hline WT entropy 10 & GMM cycle 4 \\
\hline Eigenfrequencyl 1 & Shannon energy 8 \\
\hline VFD 2 & $\operatorname{HOS} 1$ \\
\hline \multicolumn{2}{|l|}{ Feature Set 4} \\
\hline loudness 1 & transition ratiol \\
\hline spectral power 2 & stat skewness 1 \\
\hline Lyapunovexponent 1 & PoS 13 \\
\hline $\operatorname{PoS} 16$ & $\operatorname{PoS} 17$ \\
\hline PoS 18 & PoS 22 \\
\hline Shannon energy 8 & WT details 5 \\
\hline WT entropy 6 & ST map 3 \\
\hline Eigenfrequencies 14 & Eigenfrequencies2 10 \\
\hline Eigentimes 15 & HOS 6 \\
\hline HOS 13 & $G M M x 7$ \\
\hline $\begin{array}{c}\text { GMM murmur } 4 \\
\text { VFD }\end{array}$ & $V F D 3$ \\
\hline
\end{tabular}

The selected feature subsets for each feature set are listed in table I. The first set of features, feature set 1 , is reduced to 10 features after applying SFFS. The features which comprise feature set 2 in table I are the ones listed in the original work by Ahlstrom and colleagues. Since Olmez et 
al. algorithm uses all 32 features, no feature selection has been performed. Finally, the SFFS feature selection strategy was applied to all the features mentioned in the previous section. The output of SFFS on the overall 256 features is identified as feature set 4 composed by 23 features.

Training was performed with $50 \%$ of the available data in the test database. The achieved results are shown in table II.

TABLE II

MURMUR CLASSIFICATION RESULTS WITH THREE SET OF SELECTED FEATURES.

\begin{tabular}{lcrrr}
\hline \hline Sensitivity (Se in \%) & Feature sets & & & \\
\hline \hline Murmur Type & set 1 & set 2 & set 3 & set 4 \\
\hline AS & 94.02 & 91.02 & 92.10 & 93.02 \\
AR & 95.37 & 89.90 & 88.04 & 94.78 \\
MR & 90.85 & 86.43 & 93.21 & 95.20 \\
PR & 91.34 & 89.15 & 84.22 & 93.21 \\
PS & 100 & 100 & 94.33 & 100 \\
SAS + VSD & 96.17 & 95.39 & 86.71 & 90.41 \\
SE & 100 & 99.13 & 90.82 & 100 \\
\hline Overall & 95.74 & 93.02 & 90.51 & 96.15 \\
\hline \hline Specificity (Sp in \%) & Feature sets & & & \\
\hline \hline Murmur Type & set 1 & set 2 & set 3 & set 4 \\
\hline AS & 88.25 & 96.68 & 85.33 & 91.29 \\
AR & 95.49 & 95.04 & 91.63 & 93.98 \\
MR & 93.90 & 88.86 & 88.14 & 96.33 \\
PR & 96.20 & 94.83 & 89.90 & 95.80 \\
PS & 100 & 99.15 & 97.43 & 100 \\
SAS + VSD & 95.07 & 95.22 & 95.32 & 95.27 \\
SE & 100 & 100 & 98.21 & 100 \\
\hline Overall & 95.01 & 96.79 & 91.26 & 96.16 \\
\hline \hline
\end{tabular}

As can be observed, the best results have been achieved using feature set 4 . This feature set, composed by a subset of 23 features (see table I) which comprises some of the proposed new features, exhibits a $\mathrm{Se}=96.15 \%$ and $\mathrm{Sp}=96.16 \%$. These results are very similar to the results achieved with the newly proposed feature set (set 1), which obtained Se $=95.74 \%$ and $\mathrm{Sp}=95.01 \%$ with a significantly smaller set of 10 features, hence leading to a reduction in complexity. Regarding the results obtained with the feature set proposed by Ahlstrom and colleagues and Olmez and colleagues, it is observed that the former achieves similar results to the proposed strategy, although (i) a higher number (14) of features are required and (ii) a higher inter-class performance variability is observed. As for the algorithm proposed by Olmez et al. the obtained results suggest that this is the less efficient method, given that it attains the lowest results, while using the highest number of features. These results seem to suggest that (i) the proposed set of features shows evidence of adequate discriminative ability to capture the main characteristics of the most significant heart murmur classes and (ii) exhibits the overall best results in terms of trade-off between complexity and classification performance.

\section{CONCLUSIONS}

A method for heart murmur classification has been presented. The strategy followed is based on feature selection followed by a SVM classifier. A new feature set composed by 17 features to capture the time, frequency and complexity signatures of murmurs is suggested. The most discriminant subset of 10 features have been identified using sequential floating feature selection. This feature scheme with a SVM classifier was tested and compared with two well known sets of features, taken from the reference methods described in Ahlstrom et al. [8] and Olmez et al. [7], coupled to SVM classifiers.

The achieved results seem to confirm that the proposed feature set has the ability to capture the discriminative morphological and spectral characteristics of heart murmurs. Furthermore, the obtained performances confirm that the results are comparable to the results obtained using the most cited algorithms for murmur detection from heart sounds. However, these results are reached with a smaller complexity, since only 10 features are applied.

\section{ACKNOWLEDGEMENTS}

This work was performed under project SoundForLife (FCOMP-01-0124-FEDER-007243) financed by FCT (Fundação para a Ciência e Tecnologia) under program COMPETE/QREN.

\section{REFERENCES}

[1] B. Erickson, "Heart Sound And Murmurs: Across the Lifespan," in Mosby, Inc., 2003.

[2] D. Kumar, P. Carvalho, M. Antunes, J. Henriques, L. Eugenio, R. Schmidt, J. Habetha, "Wavelet Transform and Simiplicity based Heart Murmur Detection", in Proc. of 33rd Int. Conf. Computers in Cardiology, pp. 173-176, 2006.

[3] F. Rios-Gutiérrez, R. Alba-Flores, K. Ejaz, G. Nordehn, N. Andrisevic and S. Burns, "Classification of Four Types of Common Murmurs using Wavelets and a Learning Vector Quantization Network", IEEE World Congress on Computational Intelligence, pp. 2207-2213, 2006.

[4] Z. Jiang, C. Samjin, H. Wang, "A New Approach on Heart Murmurs Classification with SVM Technique", ISITC, pp. 240-244, 2007.

[5] S. L. Strunic, Fernando Rios-Gutiérrez, Rocío Alba-Flores, Glenn Nordehn, S. Bums, "Detection and Classification of Cardiac Murmurs using Segmentation Techniques and Artificial Neural Networks" CIDM, pp. 397-404, 2007.

[6] D. Ververidis and C. Kotropoulos, "Fast and Accurate Sequential Floating Forward Feature Selection with the Bayes Classifier Applied to Speech Emotion Recognition" Elsevier Signal Processing, vol. 88(12), pp. 2956-2970, 2008.

[7] T. Olmez, Z. Dokur, "Classification of Heart Sounds Using an Artifical Neural Network" Pattern Recognition Letters, pp. 617-629, 2003.

[8] C. Ahlstrom, P. Hult, P. Rask, J-E Karlsson, E. Nylander, U. Dahlstrom, P. Ask, "Feature Extraction for Systolic Heart Murmur Classification", Annals of Biomedical Engineering, Vol. 34(11), pp. 1666-1677, 2006.

[9] D. Kumar, P. Carvalho, M. Antunes, J. Henriques, L. Eugenio, R. Schmidt, J. Habetha, "Detection of S1 and S2 Heart Sounds by High Frequency Signatures", IEEE EMBS Annual International Conference, pp. 1410-1416, 2006.

[10] D. Kumar, P. Carvalho, R. Couceiro, M. Antunes, R. P. Paiva, J. Henriques, "Heart Murmur Classification using Complexity Signatures", $I C P R$, accepted, 2010.

[11] G. Tzanetakis, C. Perry, "Musical Genre Classification of Audio Signals", IEEE Tran. on Speech And Audio Processing, 10(5), pp. 293302, 2002.

[12] D. Ververidis and C. Kotropoulos, "Emotional Speech Classification using Gaussian Mixture Models and The Sequential Floating Forward Selection Algorithm", IEEE International Conference on Multimedia and Expo, pp. 1500-1503, 2005. 